ИЗВЕСТИЯ АКАДЕМИИ НАУК ЭСТОНСКОН ССР. ТОМ 25 ФИЗИКА * МАТЕМАТИКА. 1976, № 3

\title{
ОБРАБОТКА ПОЛУТОНОВЫХ ИЗОБРАЖЕНИЙ, ОСНОВАННАЯ НА ИХ ТЕКСТУРНЫХ СВОЙСТВАХ
}

Описаны алгоритмы выделения границ между областями однородной текстуры на полутоновом изображении.

\section{Введение}

Текстуры * (простые) - это особый вид изображений, которые, как правило, характеризуются:

1. Определенной структурной организацией по площади изображения.

2. Наличием большого количества более или менее одинаковых образований (элементов), создающих качественно-определенную картину.

3. Наличием доминирующих направлений расположения элементов или степенью неравномерности распределения их по площади изображения.

Отметим, что при анализе любых изображений как текстурных их следует рассматривать не поточечно, а по некоторым совокупностям точек (участкам) на изображении.

В последнее время в работах по анализу трехмерных сцен исходное полутоновое изображение часто рассматривается как текстура $\left[{ }^{1,2}\right]$. Такой подход основан на том, что объекты, которыми, например, должен манипулировать робот, имеют вид пространственной текстуры (резьба на болте, собранный узел конструкции с большим числом однотипных деталей, кучи гвоздей, шурупов и т. п.). При достаточно высокой разрешающей способности видеодатчика на изображении может быть воспроизведен микрорисунок (фактура) материала, из которого сделаны наблюдаемые объекты. Кроме того, текстурные свойства изображений можно получить с помощью специального освещения $\left[{ }^{3}\right]$. Признаки текстуры зависят также от ориентащии объектов относительно оси зрения воспринимающего устройства. Поэтому разным поверхностям объекта на изображении соответствуют более или менее однородные области с различными текстурными свойствами. Всякое изображение обладает характерной структурой, что обусловлено наличием детерминированных закономерностей в его построении. Если мы будем считать, что изображение состоит из относительно больших однородных областей

* Текстурные изображения могут быть как двухградационными, так и полутоновыми. 
(по текстуре или яркости) и границ между ними, то естественной целью обработки изображения является выделение этих областей и подчеркивание границ между ними.

Выделение областей на полутоновых изображениях по текстурным свойствам достаточно подробно описано в $\left[{ }^{4}\right]$, а путем группировки точек с одинаковой яркостью - в $\left[{ }^{5,6}\right]$. В работе $\left[{ }^{7}\right]$ участки разной текстуры выделялись методом детального анализа границ, а в работе $\left.{ }^{8}\right]$ - методом сканирования изображения в различных направлениях. Однако все предложенные в этих исследованиях способы страдают одним недостатком - они требуют детального, иногда даже поточечного анализа изображения.

Настоящую работу отличают две характерные особенности: 1) алгоритмы строятся с использованием интегральных характеристик текстуры на больших участках изображения и 2) материалом для эксперимента служит изображение объекта, автоматически сформированное системой «глаз-машина».

Процесс преобразования изображения осуществлялся нами следующим образом. Видеодатчик наводился на исследуемую сцену, после чего изображение автоматически фокусировалось с помощью ЭВМ. Затем изображение вводилось в память ЭВМ и разбивалось на прямоугольные фрагменты одинакового размера. Центры этих фрагментов образовывали однородную прямоугольную решетку. Қаждый выделенный таким образом фрагмент нелинейно дефокусировали для уменьшения локальных шумов и улучшения условий формирования текстурных признаков. Предпочтение было отдано алгоритмической дефокусировке, так как аппаратурная обладает низкой скоростью работы. Затем каждому нелинейно дефокусированному фрагменту ставили в соответствие вектор - набор интегральных характеристик текстуры, предложенный в $\left[{ }^{9}\right]$, и различными способами проводили анализ полученных векторов. Последние и определяли разные функции преобразования для выделения границ между текстурами.

В первом разделе описаны алгоритм фокусировки изображения и нелинейной дефокусировки фрагментов, применяемые интегральные характеристики текстуры и способы построения функций преобразования.

Во втором разделе кратко описан эксперимент, проведенный для проверки работоспособности предлагаемых алгоритмов. Испытания были осуществлены на системе «глаз-машина», созданной в лаборатории Д. С. Лебедева Инститvта проблем передачи информации АН СССР под руководством Г. Г. Вайнштейна. Описание этой системы можно найти в $\left[{ }^{10}\right]$.

\section{1. Алгоритмы обработки изображения}

Ав том атическая фокуси ровка. Задача оптической фокусировки сводится к установке объектива датчика изображения (телевизионной камеры) в такую позицию, чтобы на чувствительную поверхность датчика (фотокатода) проектировалось резкое изображение сцены. Выбор правильной позиции объектива весьма существен потому. что при расфокусировке изображения текстура на нем пропадает в первую очередь. Процесс фокусировки заключается в максимизации дисперсии * * на выбранном опорном фрагменте. Оценка дисперсии и является функцией резкости, величина которой зависит от положения

** Идея использования дисперсии в качестве функции резкости высказывалась А. А. Салимовым. 
объектива и имеет максимум в точке́ наибольшей резкости. Для поиска экстремума применяется алгоритм с использованием чисел Фибоначчи, который является оптимальным по числу шагов.

Алгоритм нелинейной де фокусировки. Пусть фрагмент задан отсчетами яркости $x_{j i}$ на дискретном растре $M \times N$. Вычислим:

$$
\omega=M / p, \quad \delta=N / p,
$$

где $p=5$, если на фрагменте используется три области дефокусировки. Выделение этих трех областей на фрагменте при $p=5$ проведем по следующему правилу. Отсчеты $x_{j i}$ с индексами

$$
\begin{gathered}
(1 \leqslant j \leqslant M \wedge 1 \leqslant i<\delta) \bigvee(1 \leqslant j \leqslant M \wedge 4 \delta<i \leqslant N) \vee \\
\vee(1 \leqslant j<\omega \wedge \delta \leqslant i \leqslant 4 \delta) \bigvee(4 \omega<j \leqslant M \wedge \delta \leqslant i \leqslant 4 \delta)
\end{gathered}
$$

будем считать первой областью $(\gamma=1)$, отсчеты $x_{j i}$ с индексами

$$
\begin{gathered}
(\omega \leqslant j \leqslant 4 \omega \wedge \delta \leqslant i<2 \delta) \bigvee(\omega \leqslant j \leqslant 4 \omega \wedge 3 \delta<i \leqslant 4 \delta) \bigvee \\
\vee(\omega \leqslant j<2 \omega \wedge 2 \delta \leqslant i \leqslant 3 \delta) \bigvee(3 \omega<j \leqslant 4 \omega \wedge 2 \delta \leqslant i \leqslant 3 \delta)
\end{gathered}
$$

- второй областью $(\gamma=2)$ и отсчеты $x_{j i}$ с индексами

$$
(2 \omega \leqslant j \leqslant 3 \delta \wedge 2 \delta \leqslant i \leqslant 3 \delta)
$$

- третьей областью $(\gamma=3)$.

Каждый отсчет на фрагменте преобразуем по следующему правилу:

$$
x_{j i}^{\prime \prime}=\left[\sum_{j m=j-1}^{j+1} \psi(\gamma)\left(x_{j m, i-1}+x_{j m, i+1}\right)+\psi(\gamma)\left(x_{j-1, i}+x_{j+1, i}\right)+x_{j, i}\right] / C(\gamma),
$$

где $j=2,3, \ldots, M-1 ; i=2,3, \ldots, N-1$. Коэффициенты $\psi(\gamma)$ выбираются исходя из требуемой степени дефокусировки в области, а $C(\gamma)$ - таким образом, чтобы на одинаково освещенных участках изображения отсчеты $x_{j i}$ не меняли своих значений. Для этого необходимо выполнение равенства

$$
[8 \psi(\gamma)-1] / C(\gamma)=1 .
$$

Полученные таким образом отсчеты яркостей $x_{j i}^{\prime \prime}$ будут представлять нелинейно дефокусированное изображение на фрагменте.

Из мерение параметров текстуры фрагмента. Для дальнейшего изложения целесообразно кратко напомнить процедуру вычисления интегральных признаков текстуры.

Пусть анализируемое изображение задано на дискретном растре $M \times N$. Рассмотрим центральный участок изображения размером $M^{\prime} \times N^{\prime}$, не намного отличающийся по размеру от всего изображения. Сравним его с таким же по размеру участком изображения, сдвинутым относительно исходного на расстояние $\tau$ в направлении $\varphi$. В качестве меры сходства возьмем квадрат эвклидова расстояния между векторами распределения яркостей соответствующих изображений, заданных на растре $M^{\prime} \times N^{\prime}$. Направление сдвига $\varphi$ может принимать одно из четырех значений: вправо, вправо-вверх, вверх и влево-вверх. В работе $\left[{ }^{9}\right]$ показано, что этих направлений вполне достаточно, чтобы охарактеризовать текстуру. В каждом направлении может быть осуществлено несколько разных по величине сдвигов $\tau=1,2, \ldots, K$ ( $K$ мало по сравнению с $\left.\min \left\{M^{\prime}, N^{\prime}\right\}\right)$. Значения эвклидовых расстояний для всех воз- 
можных значений $\tau$ и $\varphi$ задают $4 K$-мерный вектор $z_{R}$, который и считается вектором, характеризующим текстуру. Применение эвклидова расстояния в качестве меры сходства позволяет автоматически проводить нормировку на среднюю яркость. Если необходима нормировка по контрастности и используется дисперсия яркостей соответствующего фрагмента изображения $\sigma^{2}$, тогда вектор интегральных характеристик текстуры принимает окончательный вид

$$
z_{R}^{H}=z_{R} / \sigma^{3} .
$$

Функции преобразования текстурного изображе ния. Выберем на исходном изображении точку $Q-$ центр участка, вырезаемого окошком. Рассмотрим систему из восьми направлений $(n=8)$ : двух горизонтальных, двух вертикальных и четырех направлений по биссектрисам. Пронумеруем их $1, \ldots, n$ против часовой стрелки начиная с правого горизонтального направления. По этим направлениям на расстоянии $\mu$ расположим восемь точек и рассмотрим их в качестве центров фрагментов изображения, вырезаемых окошком. Для каждого такого участка, расположенного на расстоянии $\mu$ от центральной точки $Q$, после нелинейной дефокусировки по областям подсчитаем векторы $z_{R}^{H}$. Получим восемь (при $\left.n=8\right) 4 K$-мерных векторов. Обозначим их через $\xi(i)$, где $i=1,2, \ldots, n$. Вектор, соответствующий исходному фрагменту (центр в точке $Q$ ), обозначим через $\xi(0)$. Выражения

$$
\begin{aligned}
& F 1=\sum_{j=1}^{4 K}\left[\xi_{j}(0)-\frac{1}{n} \sum_{i=1}^{n} \xi_{j}(i)\right]^{2}, \\
& F 2=\frac{1}{n} \sum_{j=1}^{4 K} \sum_{i=1}^{n}\left[\xi_{j}(0)-\xi_{j}(i)\right]^{2},
\end{aligned}
$$

где $n=8$, назовем первой и второй функцией преобразования. Заметим, что в целях уменьшения шумов преобразования возведение в квадрат выражения, заключенного в квадратные скобки, можно заменить вычислением его абсолютной величины. Далее кратко рассмотрим принцип работы предложенных преобразований. По способу построения этих функций видно, что если точка $Q$ находится в области однородной текстуры, то $\xi(i)=\xi(0)$, где $i=1,2, \ldots, n$, и, следовательно, $F 1=0$ и $F 2=0$. На реальных изображениях получить совершенно одинаковые значения функций очень трудно (текстура квазиоднородна, имеются шумы изображения и т. д.), но в любом случае эти значения в области однородной текстуры будут малы. Однако, если какой-либо фрагмент, расположенный на расстоянии $\mu$ от точки $Q$, попадет или захватит область другой текстуры, он будет характеризоваться другим вектором текстурных признаков, отличным от остальных. При этом значения функций преобразования $F 1, F 2$ уже не будут равны нулю. Раскрыв выражения (8) и (9), можно показать, что эти функции, в силу своего построения, работают почти одинаково. Однако функция преобразования $F 1$ характеризует отличие центрального фрагмента с центром в точке $Q$ от некоторой усредненной текстуры окружения, а поэтому вычисляется на ЭВМ быстрее, чем $F 2$. Функция $F 2$ вычисляет усредненную меру сходства между центральным фрагментом и фрагментами, расположенными на расстоянии $\mu$, а поэтому дает амплитуду выделения местоположения границы между текстурами, которая в обоих случаях зависит от $\mu$, бо́льшую, чем $F 1$. 


\section{2. Эксперименты по выделению границ на полутоновом текстурном изображении}

Эксперименты проводились на сцене, представляющей собой текстурный кубик (каждая грань которого имела линейчатую текстуру). Кубик помещался перед камерой системы «глаз-машина» таким образом, чтобы три его грани занимали почти все изображение. Положение вершины кубика контролировалось по полутоновому запоминающему дисплею. Размер полного изображения сцены составлял $128 \times 128$ отсчетов яркости. Каждый отсчет кодировался шестью битами. После автоматической фокусировки изображение вводилось в память ЭВМ и разбивалось на 1156 фрагментов размером $28 \times 28$. Центры фрагментов отстояли друг от друга на четыре отсчета квантования по горизонтали и вертикали $(\mu=4)$. Таким образом, окна, которые вырезали фрагменты, были расположены с перекрытием. Нелинейная дефокусировка по областям проводилась со следующими значениями коэффициентов:

$$
\begin{array}{lll}
\gamma=1 ; & \psi(\gamma)=0,75 ; & C(\gamma)=7,0 ; \\
\gamma=2 ; & \psi(\gamma)=0,50 ; & C(\gamma)=5,0 ; \\
\gamma=3 ; & \psi(\gamma)=0,25 ; & C(\gamma)=3,0 .
\end{array}
$$

Компоненты вектора $z_{R}^{H}$-для каждого нелинейно дефокусированного фрагмента подсчитывались на растре $25 \times 25$ для четырех направлений $\varphi$. В каждом направлении осуществлялся сдвиг на 1, 2 и 3 отсчета квантования $(\tau=1,2,3)$. В итоге вычислялись 12-мерные векторапризнаки текстуры, которые обрабатывались с помощью функций преобразования $F 1$ и $F 2$. Эксперимент показал, что граница, соответствующая стыкам граней текстурного кубика, была точно выделена. Таким образом, описанный метод обработки полутоновых текстурных изображений может быть рекомендован для выделения границ между областями различной текстуры. Области однородной текстуры при использовании этого метода должны быть относительно большими по сравнению с размерами окошка, вырезающего фрагменты, а вектора, характеризующие различные текстурные области, достаточно отличаться между собой. Процесс выделения текстурной границы можно существенно ускорить, применяя методы следящей развертки, а при использовании специализированных вычислительных устройств он может протекать и в реальном масштабе времени.

\section{ЛИТЕРАТ У РА}

1. Tsuji S., Tomita F., Computer graphics and image processing, 2, 216 (1973).

2. B a jes y R., Computer graphics and image processing, 2, 118 (1973).

3. Will P. M., Pennington K. S., Artificial Intelligence, 2, 319 (1971).

4. Yegi M. G., Z avalishin N. V., Sheinin R. L., Advanced papers of the 4th intern. joint conf. on artificial intelligence, Cambrige, Massachusetts 02139, U.S.A., 1975 , p. 705 .

5. Nagao M., Hashimoto S., Sakai T., Computer graphics and image processing, 2, 272 (1973).

6. OHandley D. A., Computer graphics and image processing, 2, 281 (1973).

7. Rosenfeld A., Lee Y. H., Thomas R. B., In: Lipkin B. S., Rosenfeld A. (eds) "Picture processing and psychopictorics", New York-London, 1970, p. 381.

8. Горьян И. С., Цуккерман И. И., В сб.: Вторая Всесоюз. школа-семинар «Статистические свойства микроструктур», Тезнсы докладов, М., 1971.

9. 3 а в али шин Н. В., Мучник И. Б., Шейнин Р. Л., Автоматика и телемеханика, № 2, 95 (1975). 
10. В а йнш тейн $Г$. Г., В сб.: Роботы-манипуляторы для автоматизации ручных и вспомогательных работ, М., 1972.

\section{Институт кибернетики}

Академии наук Эстонской ССР
Поступила в редакцию

26/XII 1975

M. JOGI

\section{TEKSTUURIOMADUSTEL PŌHINEV POOLTOONILISTE KUJUTISTE TÖÖTLEMISE MEETOD}

Vaadeldakse keeruliste pooltooniliste kujutiste töötlemise algoritme, mille abil on vōimalik eristada ühesuguse tekstuuriga piirkondi. Esitatakse süsteemil silm-masin baseeruva eksperimendi tulemused.

\section{JOGI}

\section{TREATMENT OF HALF-TONE IMAGES, BASED ON THEIR TEXTURE CHARACTERISTICS}

Algorithms of the treatment of complex half-tone images with the purpose of accentuation of boundaries between the regions of homogeneous texture have been examined. The results of the experiment with the use of the system "eye - machine" are given. 